\title{
A case of dirofilaria in human eye localization
}

\author{
Cristina Lugli, Carlotta Guidetti, Luigi Vecchia \\ U.O. di Microbiologia, Arcispedale Santa Maria Nuova Reggio Emilia
}

Key words: Dirofilaria repens, microfilariae, vectors

Descrizione di un caso di dirofilariosi umana a localizzazione oculare

\section{SUMMARY}

We describe a case of infestation by Dirofilaria repens in an immunocompetent female who goes to the emergency room for a feeling irritable left eye. It requires the surgical removal of the parasite and its identification by macroscopic examination, microscopic after clarification and after fixation, paraffin embedding and staining of PAS and Grocott.

\section{INTRODUZIONE}

La dirofilariosi (o dirofilariasi) è una malattia parassitaria provocata da alcune specie di nematodi appartenenti al genere Dirofilaria. Le specie più note sono: $D$. immitis e $D$. repens (parassiti del cane e di canidi o felidi), D. ursi (dell'orso), $D$. tenuis (del procione), $D$. subdermata (del porcospino) e $D$. striata (di felidi selvatici). Tra le 27 specie note poche possono colpire l'uomo, in particolare sono riportati casi da $D$. immitis in varie parti del mondo e $D$. repens presente solo nel Vecchio Mondo. D. repens è la causa più comune di dirofilariasi. Focolai endemici di $D$. repens sono presenti in Europa meridionale ed orientale, in Asia Minore, in Asia centrale e in Sri Lanka. Apparentemente la nazione più colpita è l'Italia, con circa 300 casi pubblicati (anche se tale numero è dovuto probabilmete all'estremo interesse che il Prof. Pampiglione ha dedicato a questa parassitosi). Le dirofilarie sono trasmesse tra gli animali (ospiti definitivi) e dagli animali all'uomo da varie specie di zanzare (ospiti intermedi). In Italia, il vettore predominante è la specie Culex pipiens, ma anche Aedes albopictus, negli ultimi anni, risulta avere un ruolo importante nella trasmissione di questi parassiti. Il serbatoio naturale è rappresentato da animali domestici, canidi e felini, o selvatici, ma accidentalmente le dirofilarie possono essere trasmesse anche all'uomo nel quale provocano infestazioni di difficile diagnosi, in quanto spesso mimano altre patologie (5, 7). Le femmine di Dirofilaria generano embrioni detti microfilarie, che si trovano nel sangue dell'ospite mammifero parassitato. Le zanzare, consumando un pasto di sangue da un animale infetto assumono microfilarie che continuano il loro sviluppo nell'apparato digerente del vettore, fino a raggiungere lo stadio di larva L3. La larva L3 migra a livello della testa e va a localizzarsi nello stiletto dell'insetto, pronta per essere iniettata nell'ospite definitivo al successivo pasto di sangue del vettore ematofago $(1,2)$. Nell'ospite definitivo, le larve saranno in grado di trasformarsi in verme adulto e andranno a localizzarsi nel miocardio, nell'arteria polmonare o nel tessuto sottocutaneo a seconda delle specie (1). Il sistema immunitario dell'uomo, ospite accidentale, solitamente è in grado di bloccare lo sviluppo completo del parassita impedendo la formazione di microfilarie nel sangue, mentre il verme adulto va a localizzarsi nel sottocutaneo, a livello polmonare e in rari casi a livello congiuntivale $(1,6)$. Le zone del corpo più colpite sono gli occhi (nel tessuto sottocongiuntivale e nelle palpebre) (30.5\%), il capo (14.4\%), l'arto superiore (11.9\%) e l'arto inferiore (10.1\%). Le localizzazioni ai genitali maschili e, nella donna, alla mammella superano il 5\% dei casi rispettivamente. La dirofilariosi è descritta come una patologia in progressivo aumento in molte aree del mondo, Italia compresa (5), soprattutto a causa del surriscaldamento climatico globale, che provoca una maggior diffusione di insetti vettori, la maggior circolazione del parassita, che passa per nuove specie ematofaghe trasportatrici, l’inquinamento, lo sviluppo di resistenze verso insetticidi, la globalizzazione e gli assidui trasporti commerciali che favoriscono l'importazione di insetti vettori (4).

\section{CASO CLINICO}

Nell'Aprile del 2009, S. G. di sesso femminile, 69 anni, nata a Trapani, residente a Cavriago (RE), si presenta al Pronto Soccorso dell'Arcispedale Santa Maria Nuova di Reggio Emilia riferendo una sensazione irritativa all'occhio sinistro. All'anamnesi non risultano dati di rilievo: in famiglia non sono presenti malattie oftalmiche e la paziente non riferisce di aver effettuato recenti viaggi all'estero.

L'esame obiettivo rileva una larva raggomitolata e vitale al di sotto della congiuntiva bulbare temporale dell'occhio sinistro (Figura I) e viene fatta diagnosi di possibile congiuntivite parassitaria; l'oculomozione e i riflessi pupillari risultano normali. L'esame emocromocitometrico risulta nella norma, la ricerca di microfilarie ematiche e l'esame colturale dell'umor vitreo risultano negativi. Il parassita viene asportato dall'occhio sinistro mediante intervento chirurgico e portato al laboratorio di Microbiologia del medesimo ospedale, immerso in soluzione fisiologica. All'esame macroscopico il parassita appare filiforme, lungo circa $12 \mathrm{~cm}$, di colore bianco (Figura II). Il campione viene successivamente immerso per circa 48 ore in una soluzione di glicerolo per la chiarificazione e successivamente osservato al microscopio ottico (MO). Il parassita appare provvisto di striature longitudinali (Figura III) e di estremità arrotondate (Figura IV), e tali caratteri morfologici consentono già di avanzare la diagnosi di dirofilariosi da Dirofilaria repens. La diagnosi viene ulteriormente confermata dal laboratorio di Anatomia patologica dove il campione è stato fissato, incluso in paraffina e tagliato con il microtomo in sezioni di $6 \mu \mathrm{m}$, che sono poi state montate sul vetrino portaoggetti e colorate con le tecniche Grocott e PAS. L'osservazione in seguito a colorazione di

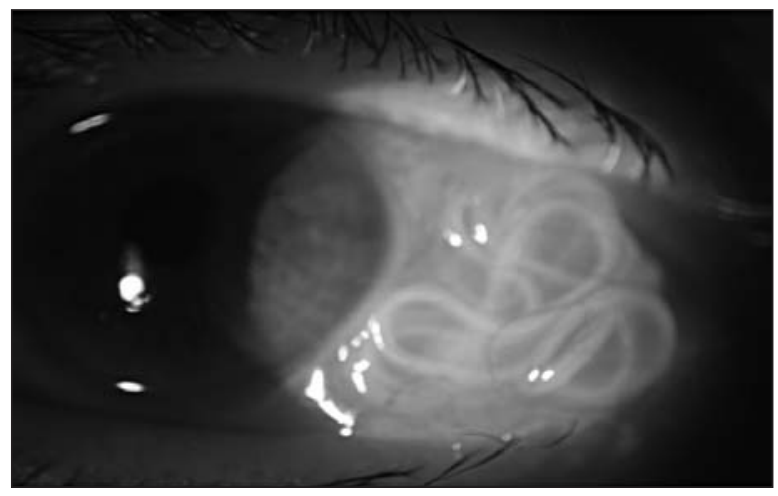

Figura I. Esemplare adulto di Dirofilaria repens in manifestazione oculare (per gentile concessione dell'unità Operativa di Oculistica Day Hospital ASMN Reggio Emilia).

\section{Corresponding author: Carlotta Guidetti}

L.P. in Microbiologia Virologia

Via Gramsci 2, 42019 Scandiano (RE) - Tel.: 3498466603

E-mail: carlottaguidetti@libero.it 
Grocott ha permesso di evidenziare la presenza di chitina (colorata in nero) e la caratteristica forma ondulata della cuticola del parassita (Figura V). La reazione di PAS, invece, ha messo in risalto la presenza di muscolatura solo ad andamento longitudinale (Figura VI).

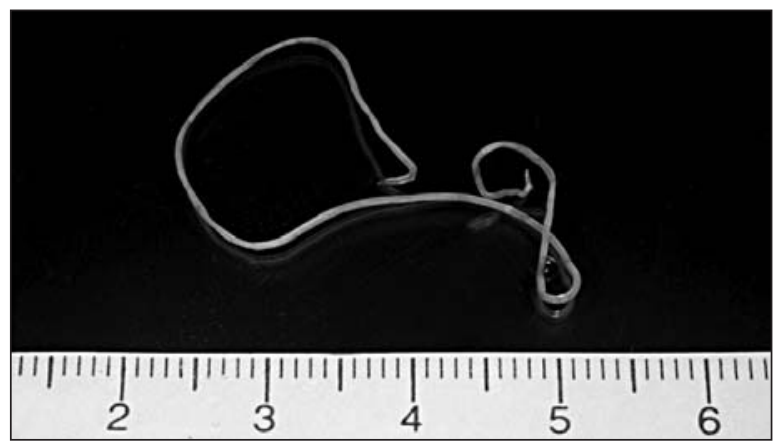

Figura II. Adulto di Dirofilaria repens (laboratorio di Microbiologia ASMN Reggio Emilia).

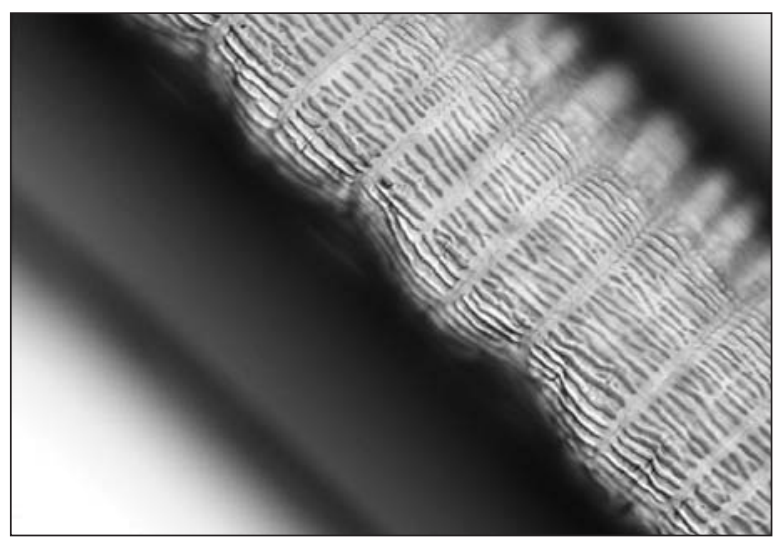

Figura III. Particolare di cuticola di Dirofilaria repens chiarificato (laboratorio di Microbiologia ASMN Reggio Emilia).

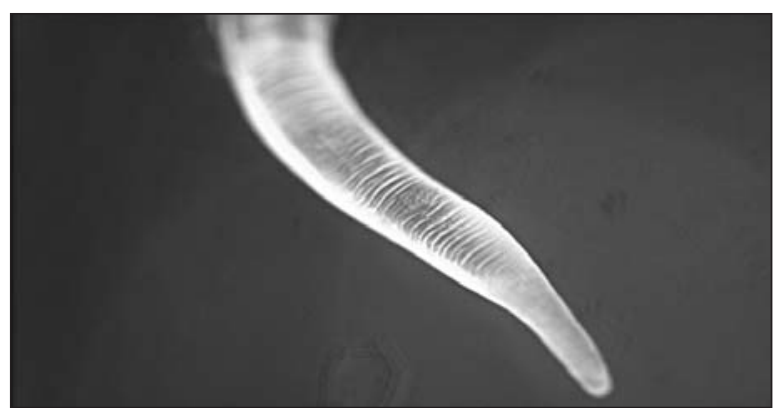

Figura IV. Parte caudale di un esemplare adulto di Dirofilaria repens chiarificato (laboratorio di Microbiologia ASMN Reggio Emilia).

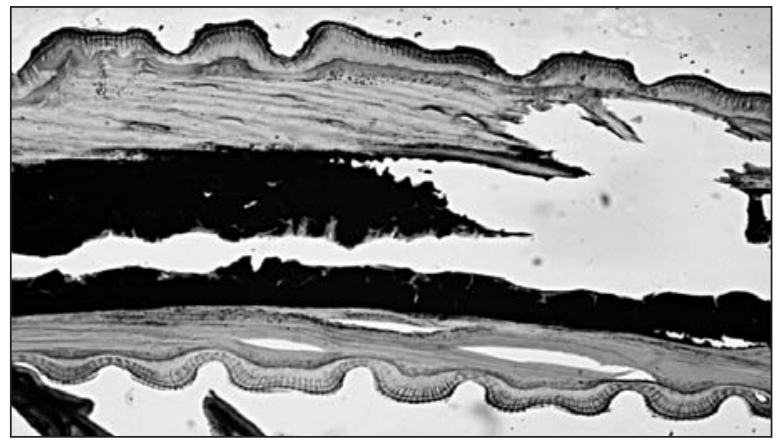

Figura V. Tecnica di Grocott di sezione longitudinale di Dirofilaria repens (laboratorio di Microbiologia ASMN Reggio Emilia).

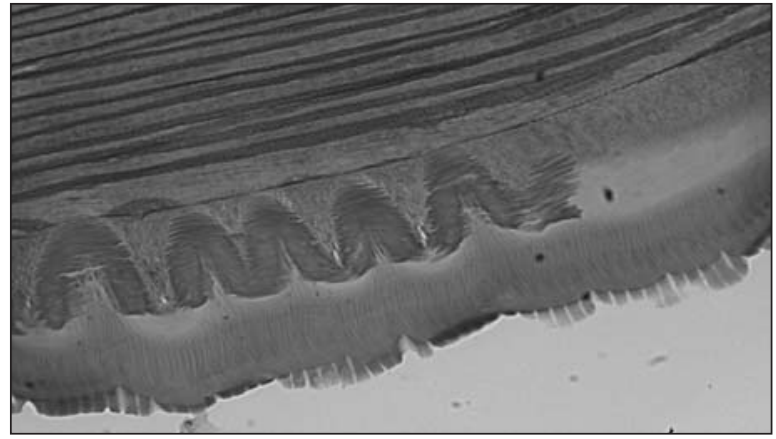

Figura VI. Colorazione di PAS di sezione longitudinale di Dirofilaria repens, (laboratorio di Microbiologia ASMN Reggio Emilia).

\section{DISCUSSIONE}

L'infestazione da Dirofilaria repens nel caso riportato, sembra verosimilmente essere stata causata dal contatto stretto con animali domestici, in quanto dall'anamnesi risulta che la paziente non aveva soggiornato in zone endemiche (Asia, Africa) e possiede un cane. Durante l'infestazione i parametri ematici non hanno mostrato segni di alterazione e successivi esami di controllo (ricerca di microfilarie nel sangue periferico negativa, rx al torace per evidenziare eventuali noduli mediastinici e ecografia bilaterale alla mammella negativi, tonometria e fondo dell'occhio nella norma), hanno permesso la dimissione della paziente senza alcuna prescrizione di terapia. La diagnosi di dirofilariosi da Dirofilaria repens riportata è la prima effettuata all'Arcispedale Santa Maria Nuova di Reggio Emilia e va ad aggiungersi agli altri 4 casi pubblicati in Emilia Romagna da Pampiglione e collaboratori relativi al periodo compreso tra il 1990-1999 (8). La dirofilariosi viene considerata una zoonosi emergente in Italia, soprattutto in relazione all'aumento globale della temperatura che favorisce l'aumento del numero di vettori, con conseguente sviluppo e diffusione dei nematodi (3). È possibile ipotizzare che l'aumento del vettore abbia fatto accrescere il numero dei cani infestati e dunque avere causato anche l'aumento di zoonosi. Vari autori $(3,8,9)$ concordano nel ritenere che l'aumento di casi di dirofilariosi ponga notevoli problemi da risolvere e richieda l'attuazione di misure per la prevenzione e la gestione della malattia. Un primo problema è quello dell'identificazione del parassita da un punto di vista medico, ma anche veterinario: la preparazione degli operatori sanitari è indispensabile per una corretta e veloce diagnosi. L’identificazione del parassita e la successiva asportazione sono fondamentali, in quanto nella maggior parte dei casi, il paziente guarisce completamente solo mediante la rimozione del nematode in particolre per le localizzazioni a livello oculare. Si sottolinea che nel caso di localizzazioni profonde in particolare se a livello polmonare, non è necessaria l'asportazione che però spesso viene fatta poiché il parassita viene scambiato per una neoplasia. L'asportazione del parassita può essere utile per la diagnosi definitiva che può essere posta solo con esame cito/istologico. La diagnosi differenziale andrebbe posta fra $D$. repens e $D$. immitis anche se, come già detto la seconda è poco frequente in Europa. Si elencano i caratteri differenziali: Dirofilaria (Dirofilaria) immitis: Maschio: cm 12-18 x um 700-900, Femmina:cm 25-30 x $\mu \mathrm{m}$ 1000-1300, cuticola liscia, senza denticolazioni, tranne che nella porzione ventrocaudale del maschio, Microfilarie: $\mu \mathrm{m}$ 290-330 x um 5-7. Dirofilaria (Nochtiella) repens: Maschio:

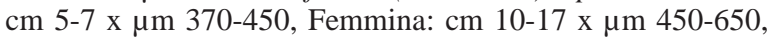
presenza di denticolazioni cuticolari longitudinali, Microfilarie $=\mu \mathrm{m} 350-380 \times \mu \mathrm{m}$ 7-8. Oltre alle figure professionali sanitarie, occorre che i possessori di cani siano ben informati riguardo la dirofilariosi, per salvaguardare il loro animale, ma anche la propria salute. Il cane deve essere periodicamente controllato dal veterinario e sottoposto a profilassi, in tal modo si preserva la salute dell'animale e si evita 
di mantenere serbatoi attivi. Altri accorgimenti che l'uomo dovrebbe adottare riguardano il vettore: per evitare lo sviluppo e la proliferazione della zanzara, e in particolar modo di A. albopictus, è necessario che non siano presenti nell'ambiente ristagni di acqua, soprattutto nel periodo estivo, mentre per non incorrere in punture di eventuali zanzare infette, l'uomo può utilizzare i repellenti in commercio, evitare di avere molte parti del corpo scoperte ed usare zanzariere e tende come barriere. L'aumento, negli ultimi anni, della diffusione di molte zoonosi rende essenziale che vengano stabilite linee guida per misure preventive, aumentino gli studi riguardanti la messa a punto di analisi sierologiche (per ora possibili presso centri di riferimento che utilizzano un antigene di dirofilarie o di Wolbachia spp) per un aiuto diagnostico, per evitare inutili interventi chirurgici anche invasivi ed una puntuale conoscenza della dirofilariosi.

\section{Ringraziamenti}

Gli autori desiderano ringraziare il Dott. Andrea Fanti, il Dott. Luca Cimino dell'Unità operativa di Oculistica e la Dott.ssa Giuliana Zoboli dell'unità operativa di Malattie Infettive dell'Arcispedale Santa Maria Nuova Reggio Emilia per la preziosa consulenza clinica.
Si ringrazia inoltre il Dott. Daniele Crotti per la generosa disponibilità nella revisione critica del manoscritto.

\section{BIBLIOGRAFIA}

1. Crespi I, Andreoni S, Bobbio F, Bruno A, Macerati R, Fortina G. Infestazione sottocutanea da Dirofiliaria spp.: descrizione di un caso clinico. Microbiologia Medica, 2007; 22 (4): 334-6.

2. De Carneri I. Parassitologia generale e umana (a cura di C. Genchi e E. Pozio). Casa Editrice Ambrosiana, Milano 2004

3. Genchi C, Rinaldi L, Mortasino M, Genchi M, Cingoli G. Climate and Dirofilaria infection in Europe. Veterinary Parasitology, 2009; 163: 28692.

4. Harrus S, Baneth G. Drivers for the emergence and reemergence of vectorborne protozoal and bacterial organisms. International Journal for Parasitology, 2005; 35: 1309-18.

5. Kramer LH, Kartashev VV, Grandi G, et al. Human Subcutaneous Dirofilariasis, Russia. Emerging Infectious Diseases, 2007; 13: 150-2.

6. Orihel TC, Eberhard ML. Zoonotic filariasis. Clin Microbiol Rev, 1998; 11: $366-81$

7. Pampiglione S, Rivasi F. Human dirofilariasis due to Dirofilaria (Nochtiella) repens: an update of world literature from 1995 to 2000 Parassitologia, 2000; 42: 231-54.

8. Pampiglione S, Rivasi F, Angeli G, et al. Dirofilariasis due to Dirofilaria repens in Italy, an emergent zoonosis: report of 60 new cases. Histopathology, 2001; 38: 344-54.

9. Pietrobelli M. Importance of Aedes albopictus in Veterinary Medicine. Parassitologia, 2008; 50: 113-5. 